\title{
1 A brain structural connectivity biomarker for diagnosis of autism 2 spectrum disorder in early childhood
}

\section{Authors}

5 Xi Jiang, $\mathrm{PhD}^{1^{*}}$, Xiao-Jing Shou, $\mathrm{PhD}^{2,3^{*}}$, Zhongbo Zhao, $\mathrm{MSc}^{1}$, Fanchao Meng, $\mathrm{MD}^{2}$, Jiao $6 \mathrm{Le}, \mathrm{PhD}^{1,5}$, Tianjia Song, $\mathrm{PhD}^{2}$, Xinjie $\mathrm{Xu}, \mathrm{PhD}^{2}$, Xiaoyan Ke, $\mathrm{MD}^{4}$, Yuzhong Chen, $\mathrm{BS}^{1}$, 7 Xiaoe Cai, MPhil ${ }^{2}$, Weihua Zhao, $\mathrm{PhD}^{1}$, Juan Kou, $\mathrm{PhD}^{1}$, Ran Huo MD ${ }^{2}$, Ying Liu MD ${ }^{6}$, 8 Huishu Yuan $\mathrm{MD}^{6}$, Yan Xing $\mathrm{MD}^{7}$, Jisheng Han, $\mathrm{BS}^{2}$, Songping Han $\mathrm{PhD}^{8}$, Yun Li, $\mathrm{MD}^{4}$, 9 Hua Lai, $\mathrm{BS}^{5}$, Lan Zhang, $\mathrm{MM}^{5}$, Meixiang Jia, BS ${ }^{9}$, Jing Liu, MD ${ }^{9}$, Keith M. Kendrick, $10 \mathrm{PhD}^{1 \#}$, Rong Zhang, $\mathrm{MD}^{2,10 \#}$

$12{ }^{1}$ The Clinical Hospital of Chengdu Brain Science Institute, MOE Key Lab for 13 Neuroinformation, University of Electronic Science and Technology of China, Chengdu, 14 611731, China.

$15{ }^{2}$ Neuroscience Research Institute; Key Laboratory for Neuroscience, Ministry of Education 16 of China; Key Laboratory for Neuroscience, National Committee of Health and Family 17 Planning of China; and Department of Neurobiology, School of Basic Medical Sciences, 18 Peking University, Beijing, 100191, China.

$19{ }^{3}$ State Key Laboratory of Cognitive Neuroscience and Learning; Beijing Key Laboratory of 20 Brain Imaging and Connectomics; and IDG/McGovern Institute for Brain Research, Beijing 21 Normal University, Beijing, 100875, China.

$22{ }^{4}$ Child Mental Health Research Center, Nanjing Brain Hospital Affiliated of Nanjing Medical 23 University, Nanjing, 210029, China.

$24{ }^{5}$ Chengdu Women's and Children's Central Hospital, School of Medicine, University of 25 Electronic Science and Technology of China, Chengdu, 611731, China.

$26{ }^{6}$ Radiology Department, Peking University Third Hospital, Beijing, 100191, China.

$27{ }^{7}$ Department of Pediatrics, Peking University Third Hospital, Beijing, 100191, China.

$28{ }^{8}$ Wuxi Shengpingxintai Medical Technology Co., Ltd., Wuxi, 214091, China.

$29{ }^{9}$ Mental Health Institute, Peking University, Key Laboratory of Ministry of Health, The 30 Ministry of Public Health, Beijing, 100191, China.

$31{ }^{10}$ Autism Research Center of Peking University Health Science Center, Beijing, 100191, 32 China 
34 *Co-first authors: Xi Jiang and Xiao-Jing Shou

\section{5 \#Joint corresponding authors:}

36 Professor Keith M. Kendrick,

37 The Clinical Hospital of Chengdu Brain Science Institute, MOE Key Laboratory for

38 Neuroinformation,

39 University of Electronic Science and Technology of China,

40 No. 2006, Xiyuan Ave, West High-Tech Zone, Chengdu, 611731, China

41 Tel/Fax: 86-28-61830811

42 E-mail: kkendrick@uestc.edu.cn

44 Dr. Rong Zhang,

45 Neuroscience Research Institute; Key Laboratory for Neuroscience, Ministry of Education of

46 China; Key Laboratory for Neuroscience, National Committee of Health and Family

47 Planning of China; Department of neurobiology, School of Basic Medical Sciences, Peking

48 University,

49 No. 38, Xueyuan Road, Haidian District, Beijing, 100191, China

50 Tel: 86-10-82801152

51 E-mail: zhangrong@bjmu.edu.cn 


\section{Abstract}

54 Objective: Autism spectrum disorder (ASD) is associated with altered brain development, 55 but it is unclear which specific structural changes may serve as potential diagnostic markers.

56 This study aimed to identify and model brain-wide differences in structural connectivity 57 using MRI diffusion tensor imaging (DTI) in young ASD and typically developing (TD) 58 children $(3 \cdot 5-6$ years old).

59 Methods: Ninety-three ASD and 26 TD children were included in a discovery dataset and 12 60 ASD and 9 TD children from different sites included as independent validation datasets. 61 Brain-wide (294 regions) structural connectivity was measured using DTI (fractional 62 anisotropy, FA) under sedation together with symptom severity and behavioral and cognitive 63 development. A connection matrix was constructed for each child for comparisons between 64 ASD and TD groups. Pattern classification was performed and the resulting model tested on 65 two independent datasets.

66 Results: Thirty-three structural connections showed increased FA in ASD compared to TD 67 children and associated with both symptom severity and general cognitive development. The 68 majority (29/33) involved the frontal lobe and comprised five different networks with 69 functional relevance to default mode, motor control, social recognition, language and reward. 70 Overall, classification accuracy is very high in the discovery dataset $96.77 \%$, and $91.67 \%$ and $7188.89 \%$ in the two independent validation datasets.

72 Conclusions: Identified structural connectivity differences primarily involving the frontal 73 cortex can very accurately distinguish individual ASD from TD children and may therefore 74 represent a robust early brain biomarker.

\section{Keywords}

77 autism; neuroimaging; diffusion tensor imaging; fractional anisotropy; brain structural 78 connectivity; diagnosis; early childhood 


\section{Introduction}

80 There is an increasing consensus that children with autism spectrum disorder (ASD) have an

81 abberant pattern of brain development. ${ }^{1-2}$ A number of structural magnetic resonance imaging 82 studies using diffusion tensor imaging (DTI) have identified altered fractional anisotropy 83 (FA), which is a widely used index in DTI to reflect axonal density and myelination, in 84 individuals with ASD, particularly in the frontal, ${ }^{3-5}$ occipital lobes, ${ }^{6}$ and corpus callosum. ${ }^{4,7}$ 85 Developmental changes in FA occur with increases in fiber tracts during the first few years, 86 followed by decreases in later childhood and adolescence, through into adulthood. ${ }^{3,5}$ 87 However, the discrimination accuracies between ASD and typically developing (TD) 88 individuals reported by a small number of studies to date following machine learning 89 classification approaches only found modest effects and have not been validated using 90 independent validation datasets $\left(75-80 \%^{6-7}\right)$. Analysis of large fiber tracts connecting many 91 different brain regions may also obscure changes in altered structural connectivity between 92 specific brain regions.

94 In the current study we have therefore used DTI to identify differences in inter-regional 95 structural connectivity at the whole-brain level in ASD compared to TD children in 294 96 different brain regions. We chose to restrict the age range of children to 3.5-6 years old since 97 this corresponds to the period when ASD symptoms have become robustly established. ${ }^{8}$ 98 Previous tractography-based research also suggests that at this age overall structural 99 connectivity differences between ASD and TD children may be less pronounced. ${ }^{4}$

101 Based on previous studies, we firstly hypothesized that ASD children would exhibit 102 significantly greater FA in structural connections at the whole brain level and particularly 103 involving frontal regions compared to TD children. Secondly, we hypothesized that altered 104 structural connections would be in networks associated with ASD symptoms and cognitive 105 and behavioral development. Finally, we hypothesized that the structural connectivity 106 changes identified would accurately predict ASD diagnosis at the individual level not only 107 within the original discovery dataset but also in two independent validation datasets.

\section{Methods}

110 Participants 
111 The present study included three independent datasets: a discovery and two validation 112 datasets.

114 Discovery dataset (Beijing). The experiment was approved by the ethics committee of the 115 Peking University Institutional Review Board (approval no. IRB00001052-13079). A total of 116119 pre-school children either diagnosed with ASD $(n=93)$ or TD children $(n=26)$ were 117 recruited. The age range of participants was 3.5 to 6 years, which is regarded as the time of 118 the most severe emerging symptoms of autism. ${ }^{8}$ Children with ASD were recruited through 119 pediatric psychiatric clinics and autism rehabilitation training centers in Beijing. Age and 120 gender matched TD children were also recruited through online social platforms or day care centers in Beijing.

ASD validation dataset (Chengdu). The experiment was approved by the ethics committee 124 of the University of Electronic Science and Technology of China (approval no. 1420190601).

125 A total of 12 ASD children were recruited aged 3 to 8 years through the child healthcare 126 department of Chengdu Women's and Children's Central Hospital.

128 TD validation dataset (Nanjing). The experiment was approved by the medical ethics 129 committee of the Brain Hospital affiliated to Nanjing Medical University (approval no. $130 \mathrm{KY} 043$ ). A total of 9 TD children were recruited aged 4 to 6 years either through the Nanjing 131 child mental health research center, online social platforms or day care centers.

133 All the participants' parents in the three datasets were informed in detail of the research 134 objectives and procedures, and provided written informed consents. Exclusion criteria were: 135 (1) neurological complications, such as epilepsy, cerebral palsy, Fragile X syndrome etc.; (2) 136 medical intervention, such as antipsychotic drugs, transcranial magnetic stimulation, 137 acupuncture etc.; (3) diagnostic imaging anomalies or craniocerebral trauma; (4) other 138 contraindications to MRI; (5) TD children had no family histories of any mental disorders 139 and exhibited no evidence of developmental delay.

\section{Clinical Diagnosis}

142 Participants in ASD groups were diagnosed at either Peking University Sixth Hospital or 143 Beijing Children's Hospital, Beijing, China for Beijing dataset, or at Chengdu Women's and 144 Children's Central Hospital, Chengdu, China for Chengdu dataset. All children in the ASD 
145 group met the diagnostic criteria of Diagnostic and Statistical Manual of Mental Disorders 146 IV-Text Revision (DSM-IV-TR) ${ }^{9}$ or Fifth Edition (DSM-5) ${ }^{10}$ and International Statistical 147 Classification of Diseases and Related Health Problems $10^{\text {th }}$ revision (ICD-10) ${ }^{11}$. In addition, 148 ASD diagnosis was confirmed in the Beijing dataset using the Autism Diagnostic 149 Observation Schedule (ADOS) ${ }^{12}$ Traditional Mandarin version, module 1 or module 2 based 150 on the child's language ability. For children in the Chengdu dataset, diagnosis was confirmed 151 using ADOS-2. ${ }^{13}$ Moreover, in the Beijing ASD and TD cohorts, cognitive ability was also 152 assessed using the Gesell Developmental Scale (GDS) ${ }^{14}$ administered by an experienced 153 pediatrician. This is a measure of cognitive and behavioral development and adaptability 154 including five components (gross motor, fine motor, adaptive, language and personal social behaviors).

\section{MRI Acquisition and Preprocessing}

158 Children in both ASD and TD groups of the three datasets were sedated by oral 159 administration of chloral hydrate at the $50 \mathrm{mg} / \mathrm{kg}$ body weight (1 $\mathrm{g}$ maximum dose), 160 commonly used for pediatric clinical imaging. During the MRI scan, children wore earplugs 161 and de-noising headsets to reduce the noise, and parents were encouraged to remain in the 162 scanning room to ensure safety in case the child awoke. In all sites, brain images were reviewed by neuroradiologists to confirm absence of neurological abnormalities.

For the discovery dataset MRI images were acquired on a GE 3T MR750 scanner with a 12channel head coil at the Peking University Third Hospital. DTI data were obtained with an echo-planar imaging sequence: $\mathrm{TR}=9,000 \mathrm{~ms}, \mathrm{TE}=89.4 \mathrm{~ms}, \mathrm{FOV}=256 \mathrm{~mm}$, matrix size $=$ $128 \times 128$, voxel size $=2 \mathrm{~mm}$ isotropic, 75 slices covering the whole brain with no gap, 32 diffusion directions, $b$-value $=1000 \mathrm{~s} / \mathrm{mm}^{2}$. For independent ASD and TD validation datasets see supplementary methods. Pre-processing methods for DTI raw data are described in the supplementary methods.

\section{Overview of analysis}

174 The flow chart of ASD identification framework is illustrated in Fig. 1. Based on the pre175 processed DTI FA map (Fig. 1(A)), the whole-brain streamline fibers were reconstructed (Fig. 176 1(B)) and adopted to the brain atlas (Fig. 1(C)). To construct the structural connection matrix 177 for each subject (Fig. 1(D)), we calculated the pair-wise FA value between every two brain 178 regions. The constructed structural connection matrix was further projected into the brain for 
179 each participant (Fig. 1(E)). Group comparisons were then performed based on all structural 180 connection networks between ASD and TD groups (Fig. 1(F)) to obtain the between-group 181 differences (Fig. 1(G)). The resulting connections were finally adopted as features to perform 182 pattern classification between ASD and TD groups (Fig. 1(H)). The details of each step are 183 demonstrated in the following sections.

\section{Construction of Structural Connection Network}

186 The brain atlas used in this study included 294 non-overlapping brain regions consisting of 187246 cortical and subcortical regions, 34 cerebellar regions, and 14 brainstem regions (Fig. 188 1(C)). The cortical and subcortical regions were from the Brainnetome Atlas ${ }^{15}$ with 123 189 homotopic regions in each hemisphere; cerebellar regions were from the Human Cerebellar 190 Probabilistic Magnetic Resonance Atlas ${ }^{16}$ and brainstem regions were from the Human 191 Brainstem Standard Neuroanatomy Atlas ${ }^{17}$. Since the three atlases were originally defined in the adult MNI152 standard space, we first performed linear registration to warp the T1weighted adult MNI152 image to the space of T1-weighted template image of children aged 4.5-8.5 years (https://www.mcgill.ca/bic/software/tools-data-analysis/anatomicalmri/atlases/nihpd), ${ }^{18}$ and then applied the linear transformation to the brain atlas to warp it to the children's template image space as well in order to obtain the young children's brain atlas.

The reconstructed whole-brain streamline fibers in each child (Fig. 1(B)) were then aligned to the young children's brain atlas space via DSI Studio ${ }^{19}$. The structural connection matrix for each child (Fig. 1(D)) was obtained by calculating the pair-wise mean FA value between every two brain regions via DSI Studio ${ }^{19}$, and further represented as a structural connection network (Fig. 1(E)) in which the nodes were the brain regions and edges were the mean FA values between two nodes.

\section{Identification of Structural Connections Showing Differences between ASD and TD}

206 Based on the structural connection networks of all children in the ASD and TD groups (Fig. 207 1(F)), we adopted the widely used Network-based Statistic (NBS) approach ${ }^{20}$ to identify the 208 structural connections and associated networks showing between-group differences (Fig. 209 1(G)). As a non-parametric statistical method, NBS first performs a large number of 210 univariate hypothesis tests on all edges in the network, then clustering-based statistics, and 211 finally permutation tests to calculate the family-wise error rate (FWER) corrected $p$-values

212 for each sub-network consisting of edges with group differences. We adopted the NBS 
213 Connectome toolbox implemented in $\mathrm{Matlab}^{20}$ to perform the analysis. The structural

214 connection networks of all participants in ASD and TD groups were the inputs with gender

215 and age as covariates. Next we performed the NBS analysis to identify both increased and

216 decreased FA values of structural connections and associated networks in ASD compared to

217 TD.

\section{Pattern Classification between ASD and TD Children}

220 Based on the identified structural connections showing between-group differences (Fig. 1(G)), 221 we further adopted FA values of those connections as features to perform pattern 222 classification between the ASD and TD groups. We used the discovery dataset as the training 223 dataset to establish the classification model. For training and leave-one-out cross-validation, 224 we employed the widely used support vector machine (SVM) approach. The training model 225 was then applied to the two independent validation datasets to validate its generalizability. 226 Specifically, we adopted the widely used cost-support vector classification (C-SVC), and 227 the radial basis function (RBF) as the kernel function in SVM. The optimal values of 228 parameters c (i.e., cost, c ranged from 15 to 16) and g (i.e., gamma, g ranged from 0.08 to 1 ) in RBF were obtained by a hyperparameter optimization framework of optuna. ${ }^{21}$

\section{Potential Effect of Imbalanced Sample Size between ASD and TD}

232 In view of the imbalanced group sample sizes in the discovery dataset (93 ASD and 26 TD),

233 we adopted both up-sampling and down-sampling approaches to alleviate the potential model 234 overfitting as well as classification bias problem (see supplementary methods). Both 235 approaches confirmed that our classification model was not influenced by the imbalanced 236 sample sizes.

\section{Statistical Analysis}

239 Independent two-sample $t$-tests were utilized to identify the different structural connections 240 between ASD and TD $(n=10,000$ permutation times, significance level $p<0 \cdot 05$, FWE 241 corrected) in NBS. Independent sample $t$-tests were used for continuous variables including 242 age, BMI, head circumference, GDS total score, and FA between ASD and TD. Chi-square 243 tests were used for categorical variables including gender and handedness between ASD and 244 TD. Pearson's linear correlation coefficients were computed between the averaged FA value 245 and ADOS and GDS scores (one-sample $t$-tests, significance level at $p<0 \cdot 05$, FDR corrected). 246 A mediation model was conducted (PROCESS) ${ }^{22}$ using bootstrap analysis to investigate the 
relationship between the averaged FA value, ADOS total and GDS total scores (bootstrap =

248 1000).

\section{Results}

\section{Subject Demographics and Behavioral Measures}

252 Table 1 summarizes demographic and other information for ASD and TD groups in the different datasets and ADOS scores for ASD children. There were no group differences in age, gender, BMI, handedness, and head circumference in the discovery dataset, although as expected the total GDS score was significantly less in the ASD group indicating impaired cognitive and behavioral development.

\section{Increased FA Connections and Networks in ASD Compared to TD Children}

We identified 33 increased but no decreased FA values of structural connections in ASD compared to TD children (Figs. 2(A) and 2(C), Table 2). Notably, 29 out of 33 connections were associated with the frontal lobe. Moreover, 30 out of 33 connections were intrahemispheric. Fig. 2(B) illustrates the locations of 33 connections on the cortical surface. In the ASD group averaged FA values were significantly negatively correlated (FDR corrected) with ADOS total and 'social interaction' sub-scale scores (Fig. 2(D)) and positively correlated with the GDS total score (Fig. 2(E)). In the TD group there was a slight but not significant negative correlation between averaged FA values and GDS score (Fig. 2(E)). A mediation analysis indicated that within the ASD group the ADOS total score was the main mediator of the effects on the averaged FA value and GDS total score (Fig. 2(F)).

270 Connections with increased FA were further categorized into 5 structural networks via NBS

271 (Fig. 2(G) and Table 2) together with a functional characterization using the Neurosynth 272 platform, and visualized as a word cloud. Network 1: default mode function and memory 273 retrieval, including: left superior temporal gyrus, precuneus, inferior parietal lobule, superior 274 frontal gyrus, and medioventral occipital cortex; Network 2: motor function, including: right 275 superior and inferior frontal gyri, right cerebellum lobe IX and left postcentral gyrus; 276 Network 3 visual and facial recognition function, including: right inferior and superior frontal 277 gyri, lateral occipital cortex, pre-motor thalamus, middle frontal gyrus, medioventral occipital 278 cortex, basal ganglia, superior temporal gyrus and insula; Network 4 language and cognitive 279 function, including: left middle and inferior frontal gyri, thalamus, cingulate gyrus, basal 
280 ganglia and cerebellum lobe IX; Network 5: social and general reward functions, including: bilateral orbitofrontal and cingulate gyri and basal ganglia.

\section{Classification Accuracy between ASD and TD Children}

284 We first up-sampled the discovery dataset to 186 subjects with 93 ASD and 93 TD and trained the classification model in a 33-dimensional feature space based on the 33 connections using a leave-one-out cross-validation strategy. Fig. 3(A) shows the classification model in a 3-dimensional feature space after performing dimensionality reduction using the $\mathrm{t}$-distributed stochastic neighbor embedding (t-SNE) algorithm. ${ }^{23}$ The Receiver Operating Characteristic (ROC) curve of the training model is shown in Fig. 3(B). The area under the ROC curve (AUC) was 0.981, indicating the robustness of the training model. The confusion matrix of the training model is shown in Fig. 3(C). Accuracy, sensitivity, specificity, precision, and $\mathrm{F}$ measure in both discovery and validation datasets are reported in Fig. 3(D) with the proposed model achieving high classification accuracy in both discovery $(96.77 \%)$ and independent validation datasets $(91.67 \%$ and $88.89 \%)$. The alternative down-sampling strategy of the discovery dataset by 1,000 times also achieved high classification accuracy in both discovery $(94 \cdot 85 \pm 1.30 \%)$ and independent validation datasets $(91 \cdot 63 \pm 5 \cdot 55 \%$ and $80 \cdot 04 \pm 5 \cdot 52 \%)$. Thus overall, the classification model showed both high accuracy and generalizability for ASD identification across different datasets without being influenced by the imbalanced sample sizes of the discovery dataset.

\section{Discussion}

302 Our findings have revealed the presence of a small number of inter-regional structural connections within the brains of young children with ASD which exhibit increased FA compared to TD and negatively associated with symptom severity. The majority of affected regional connections involve the frontal cortex and overall they achieved a classification accuracy of $96.77 \%$ for discriminating between ASD and TD individuals in the discovery dataset and $91.67 \%$ and $88.89 \%$ in two small independent datasets. The 33 inter-regional structural connections could be clustered into 5 independent networks with relevance to a range of behavioral functions influenced in ASD.

311 In support of our original hypothesis, the majority of the 33 structural connections showing

312 increased FA in children with ASD in the current study involved the frontal lobe including 
313 intrinsic short range frontal-frontal connections and longer range frontal-occipital, frontal-

314 thalamic and frontal-limbic ones. This is in agreement with findings from other studies ${ }^{3-4,24-26}$

315 and supports the conclusion that alterations in both intrinsic and extrinsic frontal lobe

316 structural connectivity contribute fundamentally to ASD.

318 The altered structural connections in children with ASD could be clustered into 5 individual 319 networks encompassing default mode, motor, visual and facial recognition, language and 320 memory and reward functions. The largest single frontal lobe cluster involved orbitofrontal 321 regions and their connections with the basal ganglia. These intrinsic frontal connections are 322 strongly associated with social and other types of reward processing as well as decision 323 making $^{27}$ and these functions are known to be impaired in ASD. ${ }^{28-29}$ Three other clusters 324 involved inferior, medial and superior frontal gyri connections with thalamus, basal ganglia, 325 cingulate, insula, occipital cortex, post-central gyrus and cerebellum associated with social 326 cognition, language comprehension, sensory and motor processing functions, ${ }^{30-32}$ all of which 327 are impaired in ASD. ${ }^{33-36}$ The remaining cluster primarily involved connections between the 328 superior temporal gyrus and inferior parietal lobule with the precuneus in the default mode 329 network, associated with self-processing, experience of agency, autobiographic and episodic 330 memory retrieval and visuospatial imagery. Default mode dysfunction has been consistently 331 reported in $\mathrm{ASD}^{37}$ as well as impaired self-processing, sense of agency, autobiographical and episodic memory. ${ }^{38-39}$

334 A previous study using DTI measures and classification techniques to identify ASD 335 compared to TD children employed shape representations of white matter fiber tracts as 336 features, and achieved $75 \cdot 34 \%$ discrimination accuracy using a leave-one-out cross-validation 337 approach. ${ }^{7}$ Another study adopted the anisotropy scores of regions of interest as features, and 338 achieved $80 \%$ accuracy using leave-one-out cross-validation. ${ }^{6}$ In our current study, we 339 adopted the DTI-derived FA values of structural connections as features, and achieved a 340 much higher classification accuracy in both the discovery dataset (96.77\%) and, importantly, 341 in independent validation datasets $(91.67 \%$ and $88.89 \%)$, demonstrating satisfying 342 classification and generalization ability of our model across different datasets.

344 Unexpectedly, we found a significant negative correlation between the averaged FA values of 345 the 33 altered connections in children with ASD and ADOS total and social sub-scale scores, 346 indicating that symptom severity was actually lower in children with greater FA. Scores on 
347 GDS were also positively correlated with FA values in the ASD group but slightly negatively 348 correlated in the TD group. A mediation analysis identified that ADOS scores were primarily 349 mediating both FA values and GDS scores in the ASD group. This may indicate an 350 experience-dependent compensatory effect is occurring in children with ASD whereby 351 increased FA contributes to reduced symptom severity and enhanced cognitive and 352 behavioral development. A social experience compensation effect has previously been 353 described in behavioral studies of autism. ${ }^{40}$ Interestingly, a tractography study has reported a 354 positive association between increased frontal lobe FA and symptom severity in very young 355 children but a negative one in older children in the age-range of the current study. ${ }^{4}$ Thus, 356 children who experience more severe symptoms at the age of 3.5-6 years may have reduced 357 FA in these neural circuits compared to when they were younger, whereas those with milder 358 symptoms may instead have maintained or even increased their FA. A longitudinal study 359 would clearly be required to confirm this possibility.

\section{$361 \quad$ Limitations}

362 A limitation of the current study is its cross-sectional nature and restricted age range $(3 \cdot 5-6$ 363 years old). Patterns of structural differences may differ in both younger and older individuals 364 and only a longitudinal design study can address this. A second limitation is we did not 365 determine whether observed changes are specific to ASD or might also occur in children with 366 developmental delay, for example. A final limitation is that we could only obtain two small 367 datasets for independent analysis of discrimination accuracy although the findings were very 368 encouraging.

\section{Conclusion}

371 By employing a fine-grained, brain-wide analysis of structural differences for regional 372 connections in the brains of young (3.5-6 years old) autistic compared with typically 373 developing children we have identified a number of structural connections mainly involving 374 the frontal lobe exhibiting increased FA but negatively associated with symptom severity. 375 Differences in these structural connections show high accuracy (>96\%) in discriminating 376 autistic children from TD children which generalizes to independent novel datasets. These 377 new findings suggest that differences in structural connections primarily involving the frontal 378 cortex of young autistic children are a potential reliable and generalizable biomarker for ASD 379 diagnosis and for assessing the efficacy of therapeutic interventions. 


\section{Contributors}

382 XJ: conceptualization, methodology, software, validation, formal analysis, resources, data 383 curation, writing - original draft, writing - review \& editing, visualization, supervision, 384 project administration, funding acquisition. XJS: methodology, validation, investigation, 385 resources, data curation, writing - review \& editing, project administration, funding 386 acquisition. ZBZ: methodology, software, validation, formal analysis, visualization. FCM: 387 investigation. JL: investigation. TJS: investigation. XJX: investigation. XYK: resources. 388 YZC: formal analysis, visualization. XEC: investigation. WHZ: methodology, formal 389 analysis. JK: investigation. RH: investigation. YL: investigation. HSY: resources. YX: 390 investigation. JSH: conceptualization, resources. SPH: investigation. YL: investigation. HL: conceptualization. LZ: conceptualization. MXJ: investigation. JL: resources. KMK: conceptualization, formal analysis, resources, writing - review \& editing, visualization, supervision, project administration, funding acquisition. RZ: conceptualization, resources,

394 writing - review \& editing, visualization, supervision, project administration, funding 395 acquisition.

\section{Declaration of interests}

397 We declare no competing interests.

\section{Data sharing}

399 Individual participant data and the data dictionary defining each field in the set will not be 400 made available as all participants did not consent to have their data as a public resource. The 401 group-level data results as well as the data processing code which do not disclose the 402 participants' information will be available with publication from the corresponding authors 403 (KMK and RZ) on reasonable request (including a research proposal), subject to review.

\section{Acknowledgments}


medRxiv preprint doi: https://doi.org/10.1101/2021.11.03.21265845; this version posted November 4, 2021. The copyright holder for this preprint

(which was not certified by peer review) is the author/funder, who has granted medRxiv a license to display the preprint in perpetuity.

It is made available under a CC-BY-NC-ND 4.0 International license .

405 This work was supported by Key Technological Projects of Guangdong Province (grant

406 number 2018B030335001 - KMK), Beijing Municipal Science \& Technology Commission

407 (grant number Z181100001518005 - RZ), the National Key Research and Development

408 Program of China (2016YFC0105501 - RZ), Sichuan Science and Technology Program

409 (2021YJ0247 - XJ), National Natural Science Foundation of China (61976045 - XJ,

41081801779 - SXJ). The funders played no role in the writing of the manuscript or the decision

411 where to submit it for publication. Furthermore, the authors would like to thank Dr. Ruo-Yan

412 Zhao for her GDS assessment. We appreciate Ms. Qing-Yun Wei of Sunshine Friendship

413 Rehabilitation Center, Beijing, China, and Ms. Meng-Lin Sun of Wucailu Rehabilitation

414 Center, Beijing, China, for their cooperation of children recruitment. Finally, we are grateful

415 to all the participant children and their families. 


\section{References}

417 1. Hazlett HC, Poe MD, Gerig G, Styner M, Chappell C, Smith RG, Vachet C, Piven J.

418 Early brain overgrowth in autism associated with an increase in cortical surface area

419 before age 2 years. Arch Gen Psychiatry. 2011; 68(5):467-76.

420 2. Girault JB, Piven J. The neurodevelopment of autism from infancy through toddlerhood.

$421 \quad$ Neuroimaging Clinical. 2020; 30(1):97-114.

422 3. Catani M, DA Flavio, B Sanja, H Henrietta, TS Michel, et al. Frontal networks in 423 adults with autism spectrum disorder. Brain. 2016; 139(2):616-30.

424 4. Solso S, Xu R, Proudfoot J, et al. Diffusion tensor imaging provides evidence of 425 possible axonal overconnectivity in frontal lobes in autism spectrum disorder toddlers. 426 Biological Psychiatry. 2016; 79(8): 676-684.

427 5. Walker L, Gozzi M, Lenroot R, et al. Diffusion tensor imaging in young children with 428 autism: biological effects and potential confounds. Biological Psychiatry. 2012; 72 : $429 \quad 1043-51$.

430 6. Ingalhalikar M, Parker D, Bloy L, et al. Diffusion based abnormality markers of 431 pathology: toward learned diagnostic prediction of ASD. Neuroimage. 2011; $432 \quad \mathbf{5 7 ( 3 ) : 9 1 8 - 2 7 . ~}$

433 7. Adluru N, Hinrichs C, Chung MK, et al. Classification in DTI using shapes of white 434 matter tracts. 2009 Annual International Conference of the IEEE Engineering in 435 Medicine and Biology Society. 2009 Sept 3-6; Minneapolis, MN, USA: IEEE; 2009 $436 \quad$ Nov. 2719 p.

437 8. Lord C, Elsabbagh M, Baird G, Veenstra-Vanderweele J. Autism spectrum disorder. 438 Lancet. 2018 Aug; 392(10146):508-20.

439 9. American Psychiatric Association. Diagnostic and statistical manual of mental 440 disorders: DSM-4. 4th edn. American Psychiantric Publishing; 2000. 
441 10. American Psychiatric Association. Diagnostic and statistical manual of mental

442 disorders: DSM-5. 5th edn. American Psychiatric Publishing; 2013.

443 11. World Health Organization. International statistical classification of diseases and 444 related health problems. 10th revision, 2nd edn. World Health Organization; 2004.

445 12. Lord C, Rutter M, Goode S, et al. Autism diagnostic observation schedule: a 446 standardized observation of communicative and social behavior. Journal of Autism and 447 Developmental Disorders. 1989; 19(2):185-212.

448 13. Lord C, Rutter M, Pamela C, et al. ADOS: Autism diagnostic observation schedule. $449 \quad$ Boston, MA: Hogrefe; 2008.

450 14. Jin X, Sun Y, Jiang F, et al. "Care for Development" intervention in rural China: a prospective follow-up study. Journal of Developmental Behavior and Pediatrics. 2007; 28(3):213-8.

15. Fan L, Li H, Zhuo J. The human brainnetome atlas: a new brain atlas based on connectional architecture. Cerebral Cortex. 2016; 26(8):3508-26.

16. Diedrichsen J, Balsters JH, Flavell J, et al. A probabilistic MR atlas of the human cerebellum. Neuroimage. 2009; 46(1):39-46.

17. Edlow BL, Takahashi E, Wu O, et al. Neuroanatomic connectivity of the human ascending arousal system critical to consciousness and its disorders. Journal of Neuropathology \& Experimental Neurology. 2012; 71(6):531-46.

460 18. Fonov V, Evans AC, Botteron K, Almli CR, McKinstry RC, Collins DL, \& Brain Development Cooperative Group. Unbiased average age-appropriate atlases for pediatric studies. NeuroImage. 2011; 54(1):313-27.

463 19. Yeh FC, Verstynen TD, Wang Y, Fernández-Miranda JC, Tseng WYI. Deterministic 464 diffusion fiber tracking improved by quantitative anisotropy. PloS One. 2013; 8(11): e80713. 
466 20. Zalesky A, Fornito A, Bullmore ET. Network-based statistic: Identifying differences in brain networks. NeuroImage. 2010; 53(4):1197-207.

21. Akiba T, Sano S, Yanase T, Ohta T, Koyama Masanori. Optuna: A next-generation hyperparameter optimization framework. Proceedings of the 25th ACM SIGKDD international conference on knowledge discovery \& data mining; Anchorage AK USA, NY USA: Association for Computing Machinery; 2019 Aug. 2623 p.

22. Hayes AF. PROCESS: A versatile computational tool for observed variable mediation, moderation, and conditional process modeling. 2012; Retrieved from https://www.afhayes.com/public/process2012.pdf

23. Laurens VDM, Hinton G. Visualizing Data using t-SNE. Journal of Machine Learning Research. 2008; 9(2605):2579-605.

24. Casanova MF. The neuropathology of autism. Brain pathology. 2007; 17(4):422-33.

25. Middleton FA, Strick PL. Cerebellar projections to the prefrontal cortex of the primate. Journal of Neuroscience. 2001; 21(2):700-12.

26. Johnson B, Stanley-Cary C, Fielding J, et al. Cerebellum and the Psychopathology of Autism and Asperger's Disorder. New York: Springer; 2014. 845 p.

27. Kringelbach ML. The human orbitofrontal cortex: linking reward to hedonic experience. Nature Reviews Neuroscience. 2005; 6:691-702.

28. Jin P, Wang Y, Li Y, et al. The fair decision-making of children and adolescents with high-functioning autism spectrum disorder from the perspective of dual-process theories. BMC Psychiatry. 2020 Apr 6; 20(1):152. autism. Autism Research. 2010; 3(2):53-67. Cognitive Science. 2018; 22(2):170-88. 
491 31. Fuster JM. The prefrontal cortex - an update: Time is of the essence. Neuron. 2011; 30:319-33.

32. Turken AU, Dronkers NF. The neural architecture of the language comprehension network: converging evidence from lesion and connectivity analyses. Front Syst Neurosci. 2011;1.

33. Harrison, L.A., Kats, A., Kilroy, E. et al. Motor and sensory features successfully

34. Kjellmer L, Hedvall A, Holm A, et al. Language comprehension in preschoolers with autism spectrum disorders without intellectual disability: Use of the Reynell Developmental Language Scales. Research in Autism Spectrum Disorders. 2012; 6(3):

35. Vlamings P, Jonkman LM, Daalen EV, et al. Basic abnormalities in visual processing affect face processing at an early age in autism spectrum disorder. Biological

36. Yi HL, Licari M, Spittle AJ, et al. Early motor function of children with autism spectrum disorder: A systematic review. Pediatrics. 2021; 147(2):e2020011270.

511 38. Souchay C, Ohlsson M, Zalla T. Autobiographical memory and theory of mind in 512 autism spectrum disorder. Atrium: WILEY Blackwell; 2018. 92 p. (Johnson JL, 513 Goodman GS, Mundy PC. The Wiley handbook of memory, autism spectrum disorder, 514 and the law; vol. 5). 
medRxiv preprint doi: https://doi.org/10.1101/2021.11.03.21265845; this version posted November 4, 2021. The copyright holder for this preprint (which was not certified by peer review) is the author/funder, who has granted medRxiv a license to display the preprint in perpetuity. It is made available under a CC-BY-NC-ND 4.0 International license.

515 39. Zhao W, Luo L, Li Q, Kendrick KM. What can psychiatric disorders tell us about 516 neural processing of the self. Front Hum Neurosci. 2013; 7:485.

517 40. Livingston LA, Shah P, Milner V, et al. Quantifying compensatory strategies in adults $518 \quad$ with and without diagnosed autism. Molecular Autism. 2020; 11:15. 
medRxiv preprint doi: https://doi.org/10.1101/2021.11.03.21265845; this version posted November 4, 2021. The copyright holder for this preprint (which was not certified by peer review) is the author/funder, who has granted medRxiv a license to display the preprint in perpetuity.

It is made available under a CC-BY-NC-ND 4.0 International license .

520 Table 1. Subject demographics and behavioral measures of the three datasets.

\begin{tabular}{|c|c|c|c|c|c|c|}
\hline & \multicolumn{2}{|r|}{ TD } & \multicolumn{2}{|r|}{ ASD } & \multirow{2}{*}{$\begin{array}{c}\text { Statistics } \\
\left(t \text { or } \chi^{2}\right)\end{array}$} & \multirow{2}{*}{$\begin{array}{c}\text { Significance } \\
(p)\end{array}$} \\
\hline & $\mathbf{N}$ & Mean (SD) & $\mathbf{N}$ & Mean (SD) & & \\
\hline \multicolumn{7}{|l|}{ Discovery (Beijing) dataset } \\
\hline Age, years & 26 & $4 \cdot 70(0 \cdot 46)$ & 93 & $4.58(0.55)$ & $-1 \cdot 07^{\mathrm{a}}$ & $0 \cdot 29$ \\
\hline Gender (boys: girls) & 26 & $20: 6$ & 93 & $85: 8$ & $2 \cdot 83^{b}$ & 0.09 \\
\hline BMI & 26 & $15 \cdot 64(1 \cdot 68)$ & 76 & $16 \cdot 03(1 \cdot 77)$ & $0 \cdot 87^{\mathrm{a}}$ & $0 \cdot 39$ \\
\hline Handedness (R: D: L) & 26 & $20: 6: 0$ & 90 & $78: 9: 3$ & $3 \cdot 76^{\mathrm{b}}$ & $0 \cdot 15$ \\
\hline Head Circumference, $\mathrm{cm}$ & 26 & $51 \cdot 24(1 \cdot 48)$ & 90 & $51.79(1.72)$ & $1 \cdot 49^{\mathrm{a}}$ & $0 \cdot 14$ \\
\hline $\begin{array}{l}\text { ADOS social interaction } \\
\text { sub-scale score }\end{array}$ & .. & .. & 86 & $9 \cdot 88(2 \cdot 54)$ & .. & .. \\
\hline $\begin{array}{l}\text { ADOS communication } \\
\text { sub-scale score }\end{array}$ & .. & .. & 86 & $5.57(1.87)$ & .. & .. \\
\hline $\begin{array}{c}\text { ADOS total score (social } \\
\text { interaction }+ \text { communication) }\end{array}$ & .. & .. & 86 & $15 \cdot 45(4 \cdot 02)$ & .. & .. \\
\hline GDS total score & 26 & $94 \cdot 39(10 \cdot 1)$ & 82 & $64 \cdot 34(17 \cdot 1)$ & $8 \cdot 50^{\mathrm{a}}$ & $<0 \cdot 001$ \\
\hline \multicolumn{7}{|l|}{ ASD validation (Chengdu) dataset } \\
\hline Age, years & .. & .. & 12 & $4.98(1.22)$ & .. & .. \\
\hline Gender (boys: girls) & .. & .. & 12 & $11: 1$ & .. & .. \\
\hline $\begin{array}{l}\text { ADOS-2 total score (social affect }+ \\
\text { restricted and repetitive behavior) }\end{array}$ & .. & .. & 12 & $18 \cdot 58(4 \cdot 46)$ & .. & .. \\
\hline \multicolumn{7}{|l|}{ TD validation (Nanjing) dataset } \\
\hline Age, years & 9 & $5.44(0 \cdot 84)$ & .. & .. & .. & .. \\
\hline Gender (boys: girls) & 9 & $6: 3$ & .. & .. & .. & .. \\
\hline
\end{tabular}

521 TD: typically developing; ASD: autism spectrum disorder; BMI: body mass index; R: right handed; D:

522 double handed; L: left handed; ADOS: Autism Diagnostic Observation Schedule; ADOS-2:

523 Second Edition of ADOS; GDS: Gesell Developmental Scale; ${ }^{a}$ Independent two-sample $t$ test, $t$

524 score; ${ }^{\mathrm{b}}$ Chi-square test, $\chi^{2}$. SD: standard deviations. 
medRxiv preprint doi: https://doi.org/10.1101/2021.11.03.21265845; this version posted November 4, 2021. The copyright holder for this preprint (which was not certified by peer review) is the author/funder, who has granted medRxiv a license to display the preprint in perpetuity.

It is made available under a CC-BY-NC-ND 4.0 International license .

526 Table 2. Profiles of the 33 structural connections with increased FA in children with ASD.

\begin{tabular}{|c|c|c|c|c|c|c|c|c|c|}
\hline \multirow{2}{*}{ No. } & \multirow{2}{*}{ Network } & \multirow{2}{*}{ Region name } & \multicolumn{3}{|c|}{ MNI coordinates } & \multirow{2}{*}{ Region name } & \multicolumn{3}{|c|}{ MNI coordinates } \\
\hline & & & $\mathbf{X}$ & $\mathbf{Y}$ & $\bar{Z}$ & & $\mathbf{X}$ & $\mathbf{Y}$ & $\mathbf{Z}$ \\
\hline 1 & \multirow{4}{*}{1} & STG_L & -55 & -3 & -10 & PCun_L & -12 & -67 & 25 \\
\hline 2 & & IPL_L & -47 & -65 & 26 & PCun_L & -12 & -67 & 25 \\
\hline 3 & & SFG_L & -18 & 24 & 53 & MVOcC_L & -13 & -68 & 12 \\
\hline 4 & & STG_L & -55 & -3 & -10 & MVOcC_L & -13 & -68 & 12 \\
\hline 5 & \multirow{3}{*}{2} & SFG_R & 20 & 4 & 64 & IFG_R & 45 & 16 & 25 \\
\hline 6 & & $\begin{array}{l}\text { SFG_R } \\
\end{array}$ & 20 & 4 & 64 & PoG_L & -21 & -35 & 68 \\
\hline 7 & & SFG_R & 20 & 4 & 64 & Right_IX & 6 & -54 & -50 \\
\hline 8 & \multirow{11}{*}{3} & IFG_R & 48 & 35 & 13 & LOcC_R & 32 & -85 & -12 \\
\hline 9 & & IFG_R & 54 & 24 & 12 & LOcC_R & 32 & -85 & -12 \\
\hline 10 & & MFG_R & 42 & 44 & 14 & MVOcC_R & 10 & -85 & -9 \\
\hline 11 & & IFG_R & 48 & 35 & 13 & MVOcC_R & 10 & -85 & -9 \\
\hline 12 & & IFG_R & 51 & 36 & -1 & MVOcC_R & 10 & -85 & -9 \\
\hline 13 & & IFG_R & 51 & 36 & -1 & BG_R & 22 & 8 & -1 \\
\hline 14 & & IFG_R & 51 & 36 & -1 & BG_R & 14 & 5 & 14 \\
\hline 15 & & IFG_R & 48 & 35 & 13 & STG_R & 47 & 12 & -20 \\
\hline 16 & & IFG_R & 54 & 24 & 12 & INS_R & 36 & 18 & 1 \\
\hline 17 & & SFG_R & 7 & -4 & 60 & Tha_R & 12 & -14 & 1 \\
\hline 18 & & IFG_R & 48 & 35 & 13 & Tha_R & 12 & -14 & 1 \\
\hline 19 & \multirow{6}{*}{4} & MFG_L & -41 & 41 & 16 & BG_L & -14 & 2 & 16 \\
\hline 20 & & MFG_L & -41 & 41 & 16 & Tha_L & -7 & -12 & 5 \\
\hline 21 & & IFG_L & -53 & 23 & 11 & BG_L & -14 & 2 & 16 \\
\hline 22 & & MFG_L & -41 & 41 & 16 & CG_R & 5 & 41 & 6 \\
\hline 23 & & MFG_L & -26 & 60 & -6 & Tha_L & -7 & -12 & 5 \\
\hline 24 & & Tha_L & -7 & -12 & 5 & Left_IX & -8 & -54 & -48 \\
\hline 25 & \multirow{9}{*}{5} & OrG_R & 23 & 36 & -18 & OrG_R & 6 & 57 & -16 \\
\hline 26 & & OrG_R & 23 & 36 & -18 & OrG_R & 9 & 20 & -19 \\
\hline 27 & & OrG_L & -6 & 52 & -19 & OrG_L & -10 & 18 & -19 \\
\hline 28 & & OrG_R & 6 & 47 & -7 & OrG_R & 9 & 20 & -19 \\
\hline 29 & & OrG_L & -7 & 54 & -7 & CG_L & -4 & 39 & -2 \\
\hline 30 & & OrG_R & 6 & 47 & -7 & CG_L & -4 & 39 & -2 \\
\hline 31 & & OrG_L & -10 & 18 & -19 & CG_L & -4 & 39 & -2 \\
\hline 32 & & OrG_R & 6 & 47 & -7 & BG_R & 15 & 14 & -2 \\
\hline 33 & & OrG_R & 6 & 57 & -16 & BG_R & 15 & 14 & -2 \\
\hline
\end{tabular}

527 Abbreviations: L, left; R, right; STG, superior temporal gyrus; PCun, precuneus; IPL, inferior parietal

528 lobule; SFG, superior frontal gyrus; MVOcC, medioventral occipital cortex; IFG, inferior frontal

529 gyrus; PoG, postcentral gyrus; LOcC, lateral occipital cortex; Tha, thalamus; MFG, middle

530 frontal gyrus; OrG, orbitofrontal gyrus; CG, cingulate gyrus; BG, basal ganglia; INS, insula. 
medRxiv preprint doi: https://doi.org/10.1101/2021.11.03.21265845; this version posted November 4, 2021. The copyright holder for this preprint (which was not certified by peer review) is the author/funder, who has granted medRxiv a license to display the preprint in perpetuity.

It is made available under a CC-BY-NC-ND 4.0 International license .

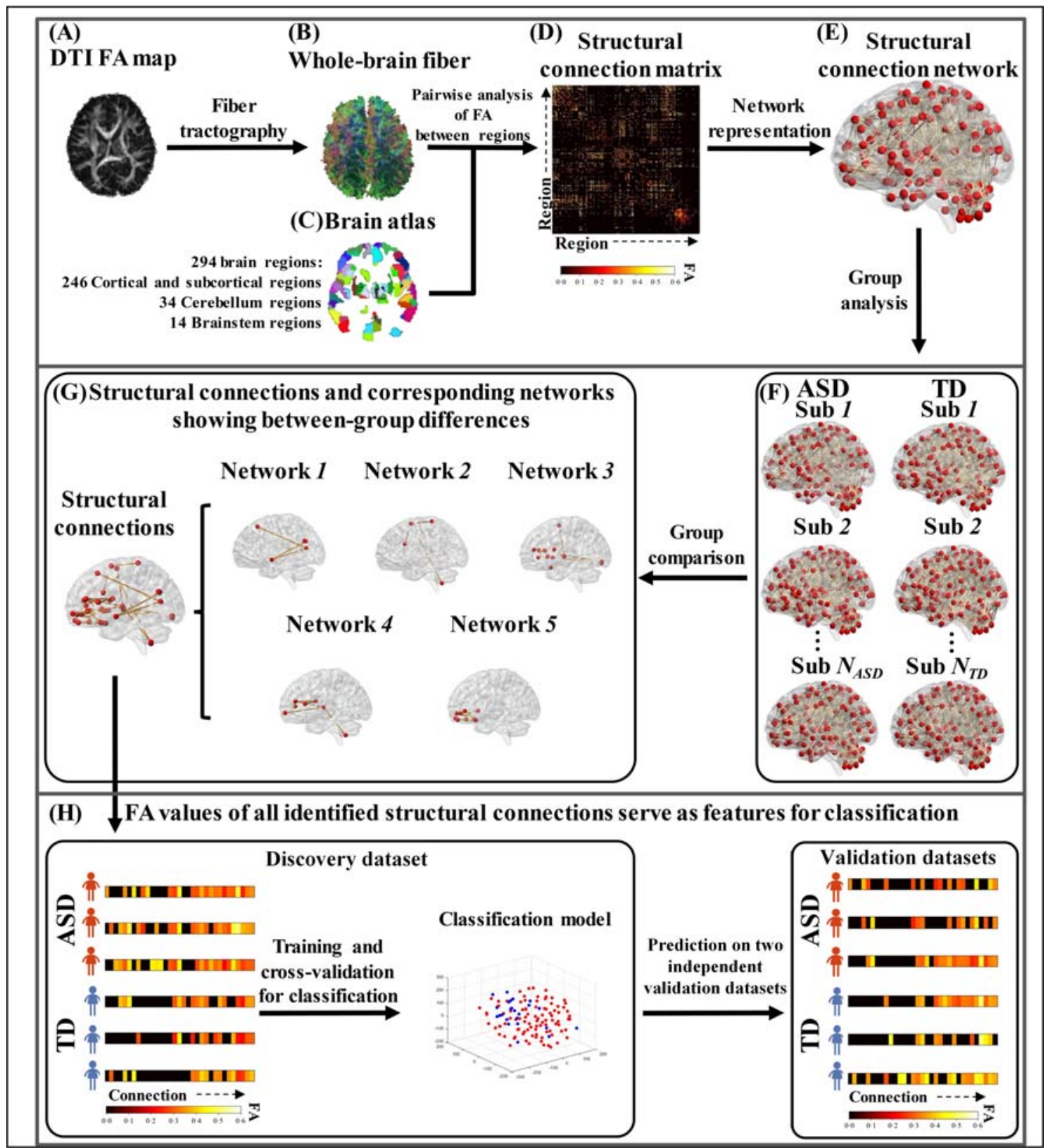

Figure 1 Flow chart of ASD identification framework. (A) The pre-processed DTI FA map. (B)

534 Reconstructed whole-brain streamline fibers. (C) Young children's brain atlas. (D) Structural

535 connection matrix for each participant. (E) The individual structural connection network. (F) All

536 individual structural connection networks in ASD and TD groups. (G) The structural connections and

537 associated networks showing differences between ASD and TD groups. (H) Pattern classification

538 between ASD and TD using all structural connections showing between-group differences. 
(A)

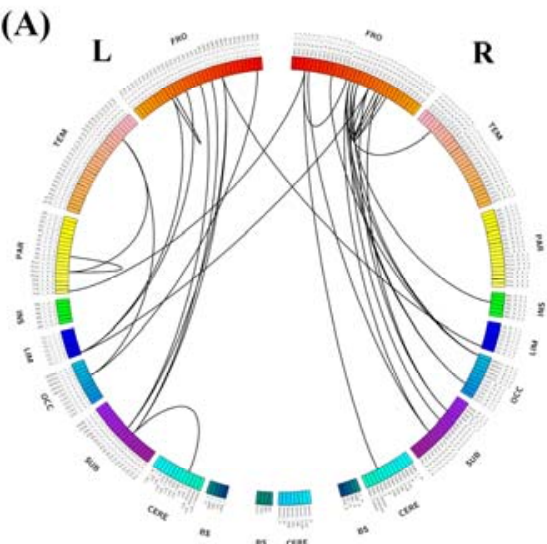

(E)

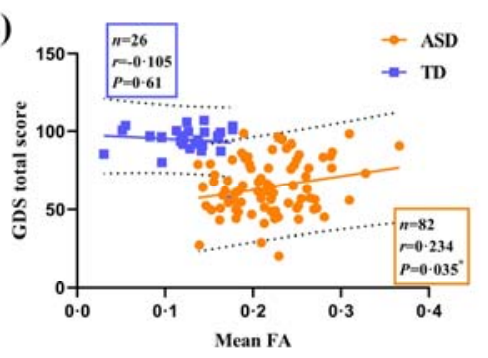

(G)

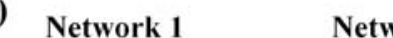

Network 2
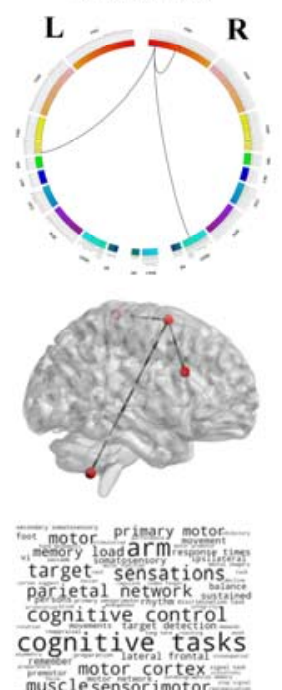

(B)

(C)
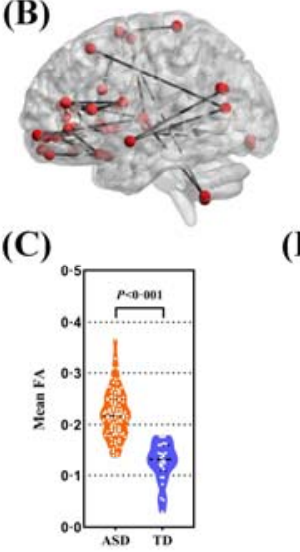

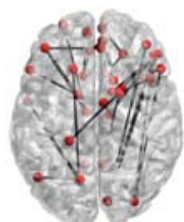

(D)

(F)
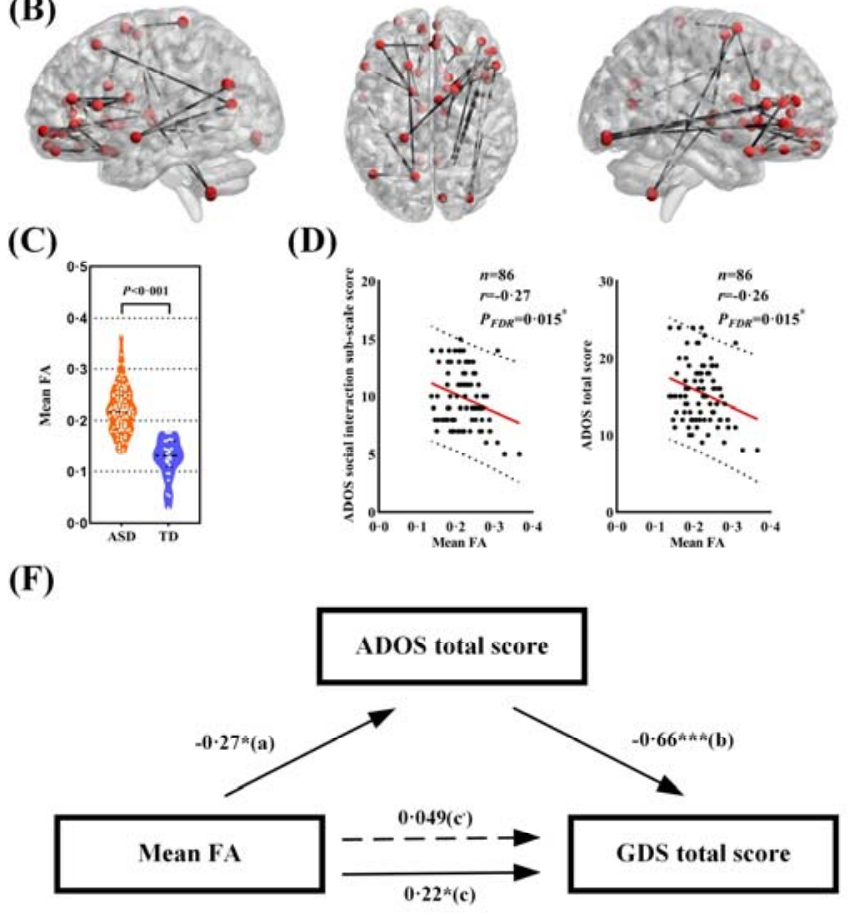
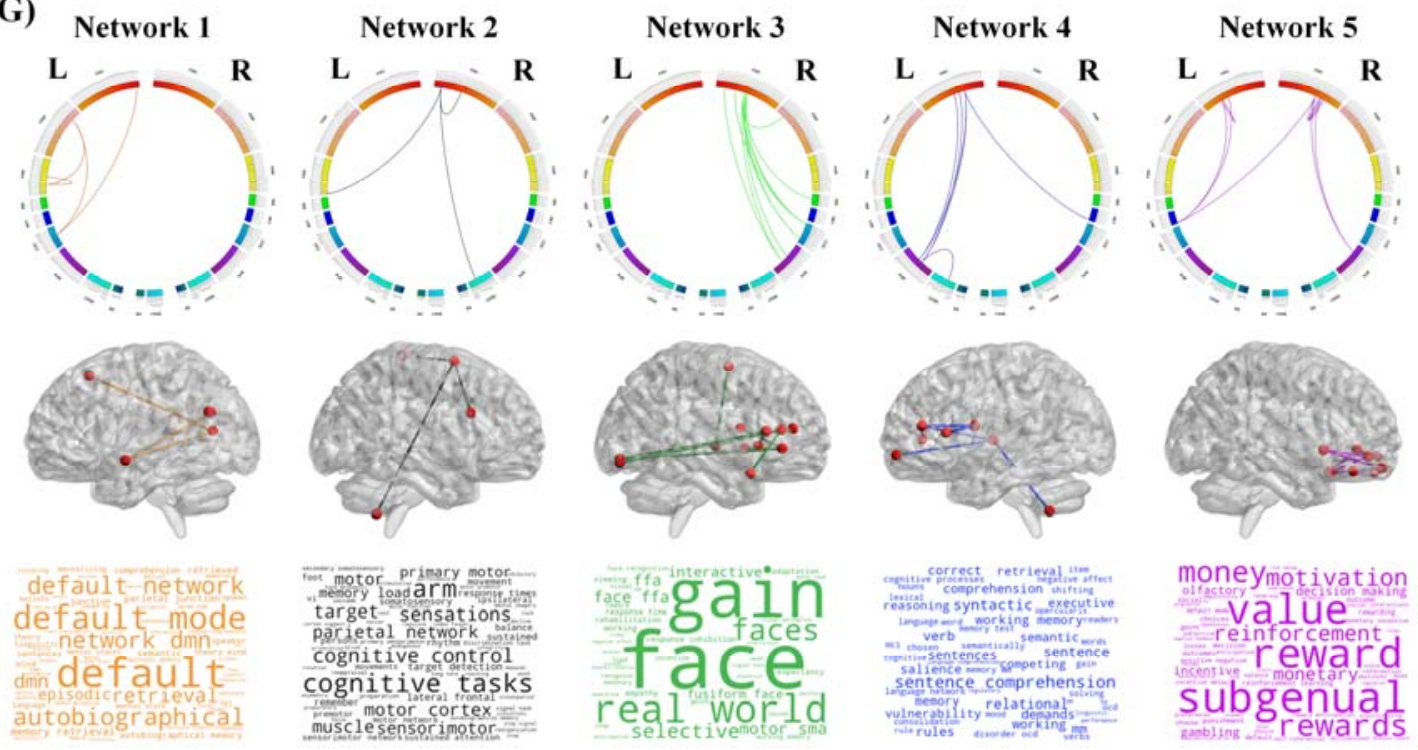

Figure 2 Increased FA connections and networks in ASD compared to TD children. (A) The 33

542 increased FA values of structural connections in ASD in circos plot. Abbreviations: L, left hemisphere;

$543 \mathrm{R}$, right hemisphere; FRO, frontal lobe; TEM, temporal lobe; PAR, parietal lobe; INS, insular lobe;

544 LIM, limbic lobe; OCC, occipital lobe; SUB, subcortical nuclei; CERE, cerebellum; BS, brain stem.

545 (B) Locations of 33 connections on the cortical surface. (C) Averaged FA value of 33 connections in

546 ASD and TD groups. (D) Correlations between averaged FA value of the 33 connections in ASD and

547 ADOS 'social interaction' sub-scale score and total ('communication' + 'social interaction') score. (E)

548 Correlations between averaged FA value of the 33 connections and ADOS social communication and

549 total scores and GDS total score. (F) Mediation analysis between averaged FA, GDS total score and 
550 ADOS total score (path $\mathrm{a}=-0 \cdot 27, p=0 \cdot 018$; path $\mathrm{b}=-0 \cdot 66, p<0 \cdot 001$; path $\mathrm{c}=0 \cdot 22, p=0 \cdot 047$; path $551 c^{\prime}=0.049, p=0 \cdot 58$ ). (G) The 33 increased connections were further categorized into 5 structural 552 networks. In each network, row 1-3 shows the structural connections in circos plot, locations of the 553 connections on cortical surface, and the word cloud of functional annotation via meta-analysis 554 respectively. 
(A)

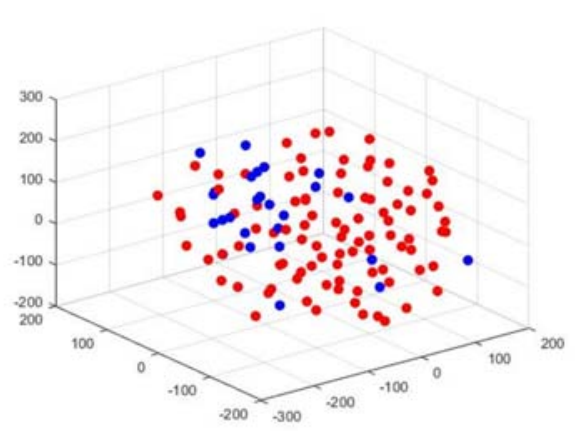

(C) Confusion Matrix

556

557

558

559

560

561

562

563
(B)

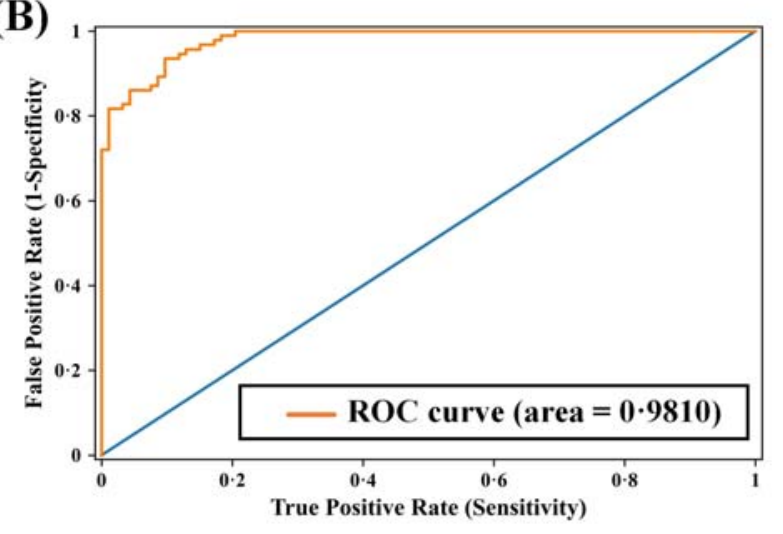

(D)

Classification Accuracy

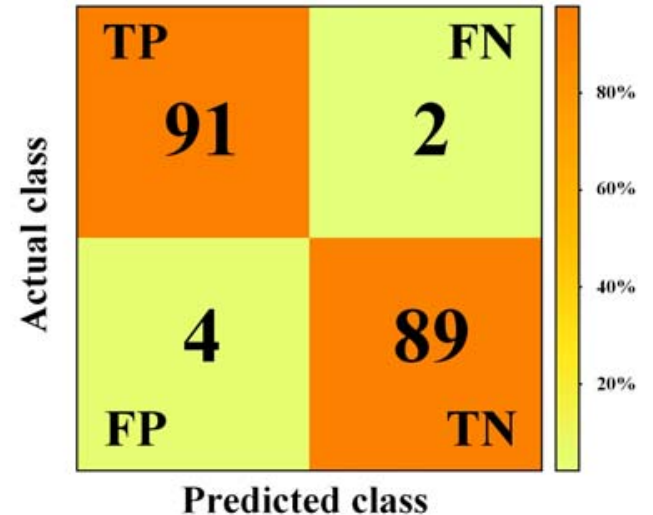

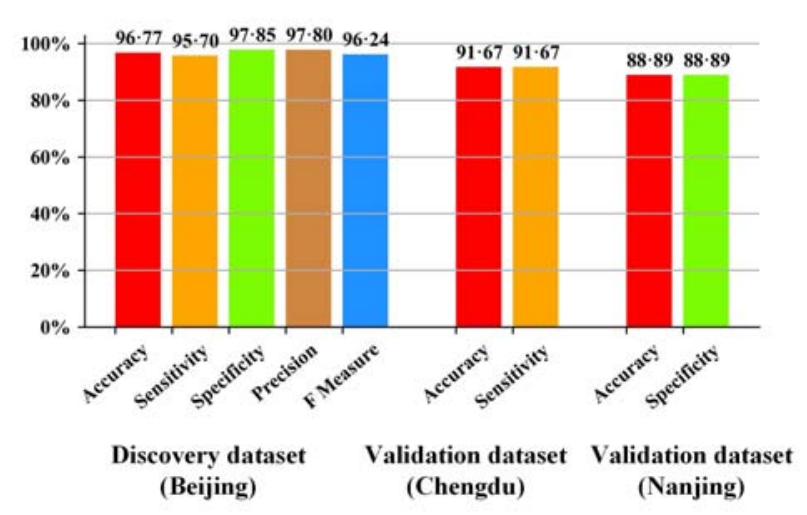

Figure 3 Classification Accuracy between ASD and TD. (A) Classification model in a 3dimensional feature space after performing dimensionality reduction using the t-distributed stochastic neighbor embedding (t-SNE) algorithm. Red and blue dots represent ASD and TD subjects, respectively. (B) The Receiver Operating Characteristic (ROC) curve of the training model. (C) The confusion matrix of the training model. The colorbar represents the proportion of correctly classified subjects among all subjects. (D) The detailed classification accuracy metrics including accuracy, sensitivity, specificity, precision, and F measure in both discovery and validation datasets. 